\title{
Delayed Capital Injections for a Risk Process with Markovian Arrivals
}

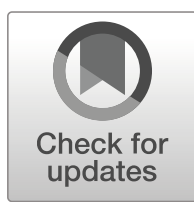

\section{A. S. Dibu ${ }^{1}$ - M. J. Jacob ${ }^{1}$ - Apostolos D. Papaioannou ${ }^{2} \cdot$ Lewis Ramsden $^{3}$ (i)}

Received: 15 April 2019 / Revised: 2 March 2020 / Accepted: 17 May 2020 /

Published online: 21 June 2020

(C) The Author(s) 2020

\begin{abstract}
In this paper we propose a generalisation to the Markov Arrival Process (MAP) risk model, by allowing for a delayed receipt of required capital injections whenever the surplus of an insurance firm is negative. Delayed capital injections often appear in practice due to the time taken for administrative and processing purposes of the funds from a third party or the shareholders of a firm. We introduce a MAP risk model that allows for capital injections to be received instantaneously, or with a random delay, depending on the amount of deficit experienced by the firm. For this model, we derive a system of Fredholm integral equations of the second kind for the Gerber-Shiu function and obtain an explicit expression (in matrix form) in terms of the Gerber-Shiu function of the MAP risk model without capital injections. In addition, we show that the expected discounted accumulated capital injections and the expected discounted overall time in red, up to the time of ruin, satisfy a similar integral equation, which can also be solved explicitly. Finally, to illustrate the applicability of our results, numerical examples are given.
\end{abstract}

Keywords Delayed capital injections - Markov Arrival Process · Systems of Fredholm integral equations · Gerber-Shiu function . Expected discounted-accumulated capital injections until ruin

Mathematics Subject Classification (2010) 45B05 - 60J25 - 91B05

Lewis Ramsden

lewis.ramsden@york.ac.uk

A. S. Dibu

dasachudham@gmail.com

M. J. Jacob

mjj@ nitc.ac.in

Apostolos D. Papaioannou

papaion@liv.ac.uk

1 Department of Mathematics, National Institute of Technology Calicut, Kozhikode, India

2 Department of Mathematical Sciences, University of Liverpool, Liverpool, UK

3 The York Management School University of York, Yorkshire, UK 


\section{Introduction}

Let $\left\{U_{t}\right\}_{t \geqslant 0}$ be the risk process of an insurer at time $t \geqslant 0$, defined by

$$
U_{t}=u+c t-\sum_{i=1}^{N_{t}} X_{i},
$$

where $u \geqslant 0$ is the insurer's initial capital, $c>0$ is the continuously received constant premium rate, $\left\{N_{t}\right\}_{t} \geqslant 0$ is a counting process denoting the number of claims received up to time $t \geqslant 0$ with a sequence of random variables $\left\{\sigma_{i}\right\}_{i \in \mathbb{N}}$ denoting the $i$-th claim epoch and $\left\{X_{k}\right\}_{k \in \mathbb{N}^{+}}$forms a sequence of i.i.d. random variables representing the amount of the $k$ th claim, having (common) cumulative distribution function (c.d.f.) $F_{X}(\cdot)$, density function $f_{X}(\cdot)$, and finite mean $\mu=\mathbb{E}(X)<\infty$.

In this paper, we consider a fairly general setting for the above risk model by assuming that the claim counting process, $\left\{N_{t}\right\}_{t \geqslant 0}$, with $\left\{\sigma_{i}\right\}_{i \in N}$ claim arrival epochs, is governed by a Markovian Arrival Process (MAP), which includes the classical and (most) Sparre Andersen risk models, as well as models with correlated inter-claim times as special cases. A MAP with representation $M A P_{m}\left(\vec{\alpha}^{\top}, \mathbf{D}_{0}, \mathbf{D}_{1}\right)$, of order $m \geqslant 1$, is a two-dimensional Markov process, denoted by $\left\{\left(N_{t}, J_{t}\right)\right\}_{t \geqslant 0}$, on the state space $\mathbb{N}_{0} \times\{1, \ldots, m\}$, where $\left\{J_{t}\right\}_{t \geqslant 0}$ denotes the state of an underlying Continuous-Time Markov Chain (CTMC), defined on the state space $E$. For $E=\{1, \ldots, m\}$, the underlying CTMC exhibits two different categories of state transition: (a) transitions between states $i$ and $j \neq i \in E$, occurring without an accompanying claim, which are given by the $(i, j)$-th element of $\mathbf{D}_{0}$, namely $D_{0}(i, j) \geq 0$, and (b) transitions from state $i$ to state $j$ (possibly with $j=i$ ) in $E$ with an accompanying claim, which are given by the $(i, j)$-th element of $\mathbf{D}_{1}$, namely $D_{1}(i, j) \geq 0$. The diagonal elements of $\mathbf{D}_{0}$ are assumed to be negative such that each row of the matrix $\mathbf{D}_{0}+\mathbf{D}_{1}$ is zero. Moreover, the initial state distribution of the underlying CTMC is given by the probability row vector $\vec{\alpha}^{\top}=\left(\alpha_{1}, \ldots, \alpha_{m}\right)$, with $\alpha_{i}=\mathbb{P}(J(0)=i)$, for $i \in E$, where $\top$ denotes the transpose vector/matrix. For more details of MAP's see Neuts (1979), Latouche and Ramaswami (1999), and Badescu et al. (2005a) and the references therein.

Due to the matrix form of the MAP set up, it has been natural throughout the literature to assume that claim amounts, $\left\{X_{k}\right\}_{k \in \mathbb{N}^{+}}$, follow a phase-type (PH) distribution, which allows for analysis of the overall risk model through the extensive theory of matrix analytic methods, see for e.g. Badescu et al. (2005a, b), Ahn and Badescu (2007), among others. In addition, given that a claim occurs during a transition from state $i$ to $j \in E$ (with rate $\left.\mathbf{D}_{1}(i, j)\right)$, the versatility of the MAP allows for the claim size distribution to be dependent on the environmental states prior to and following the claim arrival epoch, i.e. states $i, j \in E$. This flexibility provides the framework for modelling claim sizes that depend on external environmental factors, such as automobile liabilities and weather conditions or claims arising from natural disasters. Under this general formulation, any claim that occurs during a transition from state $i$ to $j \in E$ is assumed (without loss of generality, see Ramaswami (2006) pp. 501 and Appendix 2 of the same paper for details) to have a phase-type distribution of order $n \geqslant 1$, with representation $P H_{n}\left(\vec{\gamma}_{i j}^{\top}, \mathbf{H}_{i j}\right)$, which is equivalent in distribution to the absorption time of an $(n+1)$-dimensional CTMC where the transitions between its $n$ transient states are given by the transition rate matrix $\mathbf{H}_{i j}$, transitions into the absorb- 
ing state by the column vector $\vec{h}_{i j}=\left(h_{i j}(1), \ldots, h_{i j}(n)\right)^{\top}=-\mathbf{H}_{i j} \overrightarrow{1}_{n}$, where $\overrightarrow{1}_{n}$ is an $n \times 1$ column vector of units, and initial distribution given by the probability row vector $\vec{\gamma}_{i j}^{\top}=\left(\gamma_{i j}(1), \ldots, \gamma_{i j}(n)\right)$, such that the associated c.d.f. and p.d.f. are given by

$$
F_{i j}(x)=1-\vec{\gamma}_{i j}^{\top} e^{\mathbf{H}_{i j} x} \overrightarrow{1}_{n}, \quad f_{i j}(x)=\vec{\gamma}_{i j}^{\top} e^{\mathbf{H}_{i j} x} \vec{h}_{i j} .
$$

One of the first considerations of the MAP/PH model, in an insurance setting, was proposed by Badescu et al. (2005a) through its comparison with fluid queue processes. The related fluid queue process comprises of an underlying irreducible CTMC with transition intensity matrix

$$
\mathbf{T}=\left(\begin{array}{ll}
\mathbf{T}_{11} & \mathbf{T}_{12} \\
\mathbf{T}_{21} & \mathbf{T}_{22}
\end{array}\right)
$$

where, during the sojourn time that the CTMC spends in states governed by the matrix $\mathbf{T}_{11}$, the fluid process increases and, on the other hand, decreases during times spent in the states governed by the matrix $\mathbf{T}_{22}$. The connection between the fluid queue process and the risk process is realised by relating the duration of increasing fluid flow with incoming premiums - without loss of generality the premiums can be set as $c=1$ due to possibility of rescaling the time units - and, by freezing the time evolution upon a transition from increasing flow to decreasing, the decreasing fluid flow with the claim size random variables (see Badescu et al. (2005a) for details). It was pointed out in this paper that this model can incorporate dependence between claim sizes and the state of the environmental process, however, the mathematical representation was not discussed. Ramaswami (2006) extended this work and considered the dependent case more explicitly. Therefore, we will recall the construction of the sub-matrices from this work below and direct the reader to Ramaswami (2006) for further details.

In general, for the state dependent MAP/PH risk model described above, the associated fluid flow process with rate matrix $\mathbf{T}$, has $m+m^{2} n$ environmental states with sub-matrices $\mathbf{T}_{k l}$, for $k, l=1,2$, defined as follows:

(a) $\mathbf{T}_{11}=\mathbf{D}_{0}$, since any state transition that occurs without an accompanying claim is equivalent to a state transition whilst remaining in the upward flowing segment of the fluid process;

(b) In order to model the dependent claim size/downward flowing segment of the fluid process, we require three indices $(i, j, k)$, where $i, j \in E$ are the environmental states prior to and following the claim arrival epoch, respectively, and $k \in\{1, \ldots, n\}$ is the initial state of the corresponding PH claim size distribution, i.e. the state with initial probability $\gamma_{i j}(k)$. It follows that the set of all elements $(i, j, k)$ is of size $m^{2} n$ and the governing matrix $\mathbf{T}_{12}$, which describes transitions from upward flow to downward flow in the fluid process or equivalently a transition with an accompanying claim arrival, is such that the non-zero elements $\mathbf{T}_{12}(i,(i, j, k))$ are defined by

$$
\mathbf{T}_{12}(i,(i, j, k))=D_{1}(i, j) \gamma_{i j}(k),
$$

which represents the rate at which a claim arrives during a transition from state $i$ to $j \in E$ with initial state $k$ for the $\mathrm{PH}$ claim size distribution having representation $P H_{n}\left(\vec{\gamma}_{i j}^{\top}, \mathbf{H}_{i j}\right)$. 
(c) The sojourn time and state transitions whilst in the downward flowing segment of the fluid process are described by the sub-matrix $\mathbf{T}_{22}$ which has non-zero elements associated to the triple indices $(i, j, k)$, defined by the equation

$$
\mathbf{T}_{22}((i, j, r),(i, j, s))=H_{i j}(r, s), \quad \text { for } \quad 1 \leqslant r, s, \leqslant n,
$$

where $H_{i j}(r, s)$ denotes the $(r, s)$-th element of the transient matrix $H_{i j}$ for the $\mathrm{PH}$ claim size distribution. This corresponds to the possible transitions within the transient states of the PH distribution before absorption.

(d) Finally, the sub-matrix $\mathbf{T}_{21}$ contains the transition rates from the downward flow back to the upward flow in the fluid process and, due to the aforementioned connection between the fluid and risk process, is equivalent to the absorption rates of the $\mathrm{PH}$ distribution. Thus, all the non-zero elements are defined by the equation

$$
\mathbf{T}_{21}((i, j, r), j)=-\sum_{s \in\{1, \ldots, n\}} \mathbf{H}_{i j}(r, s)=h_{i j}(r) .
$$

We point out here that although there may be multiple state transitions within the 'downward flowing'/claim size segment of the process, we have frozen time at the claim arrival epoch. With this in mind, it is vital that the above set-up for the submatrices of $\mathbf{T}$ are constructed in such a way as to preserve the state of the MAP at the time of the claim arrival. That is, given that a claim occurs during a transition from states $i$ to $j \in E$, the transition matrix $\mathbf{T}$ must be constructed so that following the claim size/downward flow transitions, we arrive back in state $j$ of $\mathbf{T}_{11}=\mathbf{D}_{0}$, at which point time restarts and the risk/fluid process continues.

Remark 1 Due to the high dimensionality of the dependent model and the complexity of the possible state transitions, it is difficult to define explicit forms of the sub-matrices of $\mathbf{T}$ and thus, we have to express the matrices as described above. However, in the case of independent and identically distributed claims, i.e. no dependence on the states prior to and following the claim arrival epoch, the dimensions of $\mathbf{T}$ reduce to $m+m n$ and the submatrices can be written explicitly as

$$
\mathbf{T}=\left(\begin{array}{ll}
\mathbf{T}_{11} & \mathbf{T}_{12} \\
\mathbf{T}_{21} & \mathbf{T}_{22}
\end{array}\right)=\left(\begin{array}{cc}
\mathbf{D}_{0} & \vec{\gamma}^{\top} \otimes \mathbf{D}_{1} \\
\vec{h} \otimes \mathbf{I}_{m} & \mathbf{H} \otimes \mathbf{I}_{m}
\end{array}\right),
$$

where $\mathbf{I}_{m}$ denotes the $m$-dimensional identity matrix, $\otimes$ the Kronecker product and $\vec{\gamma}^{\top}, \mathbf{H}$ and $\vec{h}$ represent the (common) PH claim size distribution of order $n$ (see Badescu et al. 2005a). Moreover, in the non-dependent, we can express the so-called net profit condition by

$$
\left[\vec{\pi}^{\top} \mathbf{D}_{1} \overrightarrow{1}_{m}\right]^{-1}>-\left[\vec{\gamma}^{\top} \mathbf{H}^{-1} \overrightarrow{1}_{n}\right]
$$

where $\vec{\pi}^{\top}$ denotes the stationary probability of the CTMC, $\left\{J_{t}\right\}_{t} \geqslant 0$. This can be modified for the dependent case by accommodating for the variety of different $\mathrm{PH}$ transient generators $H_{i j}$ and and corresponding initial vectors $\vec{\gamma}_{i j}^{\top}$, the details of which are omitted here.

For the above MAP/PH model we define $T=\inf \left\{t>0: U_{t}<0\right\}$ to be the time to ruin and

$$
\phi_{\delta}(u)=\sum_{i=1}^{m} \alpha_{i} \phi_{\delta, i}(u),
$$

where

$$
\phi_{\delta, i}(u)=\mathbb{E}\left(e^{-\delta T} \omega\left(U_{T}-,\left|U_{T}\right|\right) \mathbb{I}_{(T<\infty)} \mid U_{0}=u, J_{0}=i\right)
$$


to be the Gerber-Shiu expected discounted penalty function, where $\delta \geqslant 0$ is a discount factor, $\omega(\cdot, \cdot)$ a non-negative, bivariate function describing the penalty at ruin, $U_{T-}$ and $\left|U_{T}\right|$ the surplus immediately prior and deficit at ruin, respectively, and $\mathbb{I}_{(A)}$ denotes the indicator function with respect to the event $A$.

The Gerber-Shiu function was first introduced in the seminal paper of Gerber and Shiu (1998) and unifies a variety of different risk quantities into a single function; a detailed study of which, for the MAP/PH risk model, can be found in Ahn and Badescu (2007). In the following years, the MAP risk model has attracted some attention in the actuarial and queuing theory literature and has been extended beyond the consideration of this general risk quantity in a number of different directions, see Badescu et al. (2007b) for a risk model with a multi-threshold strategy, Dibu and Jacob (2019) for a claim/by-claim risk model, Cheung and Landriault (2009) for the perturbed MAP risk models with dividend barrier strategies and the references therein.

On the other hand, as well as technical extensions to distributional aspects of the classic risk model, there have also been significant generalisations in terms of market features. In particular, the consideration of possible capital injections to restore the capital level when a firm is in deficit. The addition of capital injections within the classic risk model was first proposed by Pafumi (1998), for which a fall below zero required a necessary capital injection, equal to the size of the deficit below zero, to restore the surplus. Following its inception, the capital injection risk model has received a great deal of attention [see for example (Kulenko and Schmidli 2008; Nie et al. 2011) and the references therein], with all of models considering instantaneous receipt of the required injection.

In practice, capital injections are usually applied to a firm following some delay time (due to administration or processing requirements or periodicity of the decision for the capital injection - for the latter see Zhang et. 2018) and thus, the instantaneous receipt of capital injections proposed in the current models is, in some cases, an unnatural consideration. Ramsden and Papaioannou (2019) considered the Cramér-Lundberg risk model with delayed capital injections and derived a number of results for the ruin probability, however, their assumption that there is no restriction on the maximum size of the required capital injection and thus, the possibility to recover from an arbitrarily large deficit, is optimistic. In this paper, in order to better capture the reality of capital injection transactions, we introduce a delayed capital injection risk model with deficit constraints that allow for correlations between inter-claim times and claim amounts. For this model, we show that the Gerber-Shiu function satisfies a system of Fredholm integral equations, which can be solved to provide an explicit solution.

This paper is organised as follows: In Section 2, we define a MAP/PH risk models with delayed capital injections. In Section 3, we derive a system of Fredholm integral equations for the Gerber-Shiu function. The latter system of integral equations is solved explicitly in Section 4. In Section 5 , we consider a further quantity of interest, namely the expected accumulated capital injections up to time of ultimate ruin and show that these quantities also satisfy the Fredholm integral equation of the previous sections, which can be solved explicitly. Finally, in Section 6, we give numerical examples to illustrate the applicability of the results.

\section{The Delayed Capital Injection Risk Model}

In this section, we will introduce a modified form of the delayed capital injection risk model proposed by Ramsden and Papaioannou (2019), adapted to the MAP/PH setting and define the associated time to ultimate ruin. 
Firstly, in a similar way to Ramsden and Papaioannou (2019), let us consider a deterministic value $k \geqslant 0$, which, in the following, will be referred to as a critical value for the deficit of the surplus process, indicating whether the receipt of a necessary capital injection comes with some time delay or is received instantaneously. Further, let us impose a second critical value, denoted by $\ell \geqslant k \geqslant 0$, such that a deficit greater than this level results in immediate ruin. Then, due to the fact that the (possible) delay time of a capital injection is dependent on the deficit size at the first deficit time, $T$, we have three different possibilities:

(i) $\left|U_{T}\right| \leqslant k$, for which a capital injection of size $\left|U_{T}\right|$ is required to restore the surplus to a non-negative level and is received instantaneously from readily available funds;

(ii) $k<\left|U_{T}\right|<\ell$, for which the available funds are unable to cover the required capital injection and thus, the injection is received after some random time delay, distributed according to the (common) continuous random variable $L$, with c.d.f. $F_{L}(\cdot)$ and p.d.f. $f_{L}(\cdot)$. During this delay time of the capital injection, we assume that the company continues to receive premium income and thus, if the surplus is able to recover to the zero level exclusively from these premiums before the receipt of the capital injection, and the occurrence of subsequent claim (in which case ruin occurs - see below), the company cancels the processing capital injection and restarts from this point [see Fig. 1b].

(iii) $\left|U_{T}\right| \geq \ell$, for which the shareholders are not willing to inject the required amount of capital in a single transaction and immediate ruin occurs at this point [see Fig. 1d].

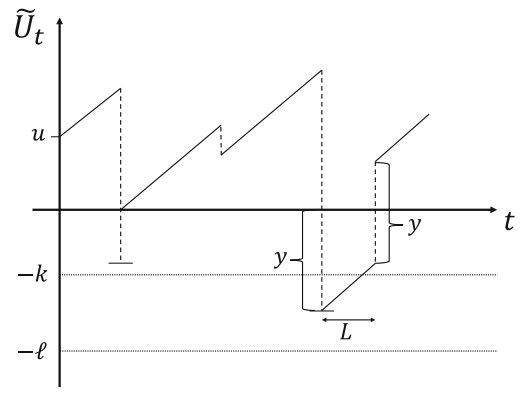

(a) Delayed capital injection arriving before subsequent claim in deficit.

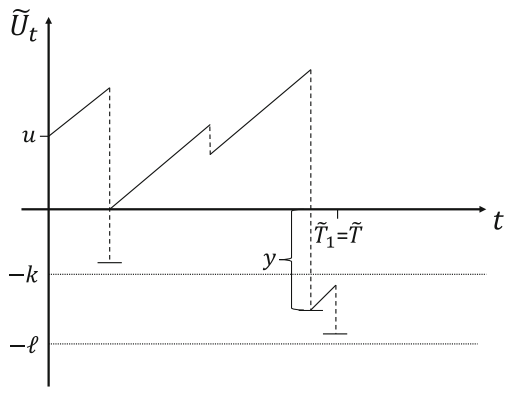

(c) Subsequent claim arriving before delayed capital injection, resulting in ultimate ruin.

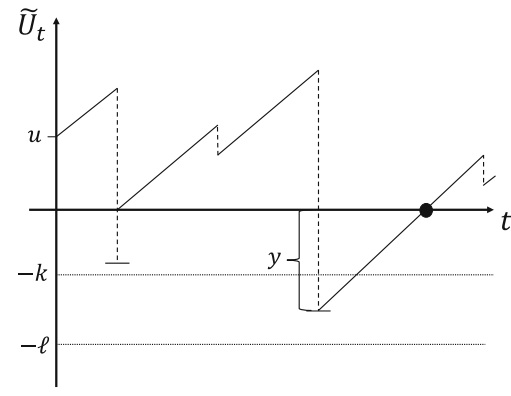

(b) Cancellation of capital injection following recovery by premiums.

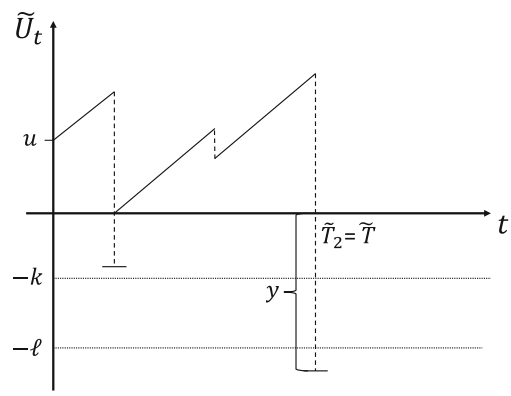

(d) Deficit larger than second critical level $-\ell$, resulting in immediate ruin.

Fig. 1 Possible sample paths following a fall into deficit 
Given the above set up, the modified surplus process with delayed capital injections, denoted $\left\{\widetilde{U}_{t}\right\}_{t \geq 0}$, is given by

$$
\widetilde{U}_{t}=U_{t}+\sum_{i=1}^{\infty}\left|\widetilde{U}_{T_{i}}\right| \mathbb{I}_{\left(\left\{\left|\widetilde{U}_{T_{i}}\right| \leqslant k\right\} \cup\left\{\left(k<\left|\widetilde{U}_{T_{i}}\right|<\ell\right) \cap\left(T_{i}+L_{i} \leqslant t\right)\right\}\right)},
$$

where $T_{i}=\inf \left\{t>T_{i-1}: \widetilde{U}_{t}<0, \widetilde{U}_{t-} \geqslant 0\right\}$, is the $i$-th time the surplus falls below zero, with $T_{0}=0$ and $T_{1}=T$, and $\left\{L_{i}\right\}_{i \in \mathbb{N}^{+}}$is the sequence of delay time random variables corresponding to the $i$-th deficit, with $\mathbb{P}\left(L_{i}=0\right)=1$ almost surely if $\left|\widetilde{U}_{T_{i}}\right| \leqslant k$ and $L_{i} \stackrel{d}{\sim} L$, otherwise.

For the modified process $\left\{\widetilde{U}_{t}\right\}_{t \geq 0}$, ultimate ruin can occur in one of two ways such that the time to ruin, denoted $\widetilde{T}$, is defined by

$$
\widetilde{T}=\min \left\{\widetilde{T}_{1}, \widetilde{T}_{2}\right\}
$$

where

$$
\widetilde{T}_{1}=\inf \left\{\sigma_{i}>0:-\ell<\widetilde{U}_{\sigma_{i-1}}<-k, \sigma_{i}-\sigma_{i-1}<\left\{L_{j} \wedge\left|\widetilde{U}_{\sigma_{i-1}}\right|\right\}\right\},
$$

for $i, j \in \mathbb{N}^{+}$, is the time of ruin due to the occurrence of a subsequent, following a fall into deficit between $-\ell$ and $-k$, before the receipt of the delayed capital injection or recovery to zero via premiums alone [see Fig. 1c] and

$$
\widetilde{T}_{2}=\inf \left\{t \geqslant 0: \widetilde{U}_{t} \leqslant-\ell\right\}
$$

is the time of ruin due to a claim occurrence that drops the surplus below the level $-\ell$ [see Fig. 1d]. Thus, the corresponding Gerber-Shiu function for the risk model in Eq. 2.1, denoted $\widetilde{\phi}_{\delta, k, \ell}(u) \equiv \widetilde{\phi}_{\delta}(u)$, is defined by

$$
\widetilde{\phi}_{\delta}(u)=\sum_{i=1}^{m} \alpha_{i} \widetilde{\phi}_{\delta, i}(u)
$$

where

$$
\left.\widetilde{\phi}_{\delta, i}(u)=\mathbb{E}\left(e^{-\delta \widetilde{T}} \widetilde{\omega}\left(\widetilde{U}_{\widetilde{T}_{-}}, \mid \widetilde{U}_{\widetilde{T}}\right) \mid\right) \mathbb{I}_{(\widetilde{T}<\infty)} \mid \widetilde{U}_{0}=u, J_{0}=i\right), \quad \text { for } \quad i \in E .
$$

Remark 2 Due to the set up of this model and definition of ultimate ruin, the surplus prior to ruin can, in fact, be negative and be interpreted as the deficit prior to ruin. This extends the classical Gerber-Shiu function for which the penalty function takes only positive variables.

The results for the Gerber-Shiu function defined in Eq. 2.2 will be given in terms of risk quantities of the MAP/PH risk model in the absence of capital injections. For this reason, we further define the following associated risk quantities, for which explicit expressions can be found in the MAP/PH risk model and can be easily retrieved as special cases of the Gerber-Shiu function, see for e.g. Ahn and Badescu (2007).

Given that the MAP process has initial state $i \in E$ and is in state $j \in E$ at the time of deficit, let $\mathbf{F}^{[a, b]}(u, t, x)=\left(F_{i j}^{[a, b]}(u, t, x)\right)_{i, j=1}^{m}$ with elements

$$
F_{i j}^{[a, b]}(u, t, x)=\mathbb{P}\left(T<t, U_{T-} \leqslant x, a \leqslant\left|U_{T}\right| \leqslant b, J_{T}=j \mid J_{0}=i, U_{0}=u\right),
$$


be the joint probability of the time to deficit, the surplus prior to deficit and the size of the deficit in the interval $[a, b]$. Then, we denote the corresponding joint density matrix by

$$
\mathbf{F}^{\prime}(u, t, x, y)=\left(f_{i j}(u, t, x, y)\right)_{i, j=1}^{m}=\left(\frac{\partial^{3}}{\partial t \partial x \partial y} F_{i j}^{[a, y]}(u, t, x)\right)_{i, j=1}^{m}
$$

Finally, let $\mathbf{W}^{[a, b]}(u, t)=\left(W_{i j}^{[a, b]}(u, t)\right)_{i, j=1}^{m}=\lim _{x \rightarrow \infty} \mathbf{F}^{[a, b]}(u, t, x)$ denote the $m$ dimensional square matrix, whose elements represents the joint probability of the time to deficit and the size of a deficit in the interval $[a, b]$, with corresponding joint density matrix

$$
\mathbf{W}^{\prime}(u, t, y)=\left(w_{i j}(u, t, y)\right)_{i, j=1}^{m}=\left(\int_{0}^{\infty} f_{i j}(u, t, x, y) d x\right)_{i, j=1}^{m}=\left(\frac{\partial^{2}}{\partial t \partial y} W_{i j}^{[a, y]}(u, t)\right)_{i, j=1}^{m}
$$

\section{A Matrix Fredholm Integral Equation}

In this section, we use probabilistic arguments to show that the Gerber-Shiu function vector $\overrightarrow{\boldsymbol{\Phi}}_{\delta}(u)=\left(\widetilde{\phi}_{\delta, 1}(u), \ldots, \widetilde{\phi}_{\delta, m}(u)\right)^{\top}$, satisfies a Fredholm integral equation of the second kind.

Due to the number of possible events that can occur following a fall into deficit, we separate the events into individual cases. That is, by conditioning on the size of the deficit below zero, the subsequent claim inter-arrival time and corresponding capital injection delay time, $L$, we have the following cases:

(i) A deficit of at most $k \geqslant 0$ : In this case, the capital injection is received instantaneously and the MAP process restarts with zero surplus;

(ii) A deficit between the two critical levels $k \geqslant 0$ and $\ell \geqslant k$ : In this case, the capital injection is received after some time delay during which there exists three further possible events:

(a) The capital injection is received before the arrival of a subsequent claim or recovery to the zero level via premiums and the process restarts from some positive capital. Note that, during the time delay the MAP may make non-claim state transitions which are described by the matrix exponential $e^{\mathbf{D}_{0} s}$;

(b) A claim arrival occurs before the capital injection is received or the process recovers to the zero level via premiums. In this case, ultimate ruin occurs $\left(\widetilde{T}_{1}\right)$ and a penalty $\widetilde{\omega}(x, y)$ is enforced ;

(c) Neither the capital injection nor a claim arrival occurs before the surplus is able to recover to the zero level via premiums. Thus, the capital injection is cancelled and the surplus restarts from zero, accounting for any (possible) non-claim state transitions of the MAP;

(iii) Finally, the deficit can exceed the critical level $\ell \geqslant k \geqslant 0$. In this case, immediate ruin occurs $\left(\widetilde{T}_{2}\right)$ and a penalty $\widetilde{\omega}(x, y)$ is enforced.

Hence, by conditioning on the time of the possible events described above (in order), the state of the MAP at the time of deficit and using the joint distribution matrices defined in 
Section 2, it follows that the Gerber-Shiu function vector, $\overrightarrow{\boldsymbol{\Phi}}_{\delta}(u)$, satisfies the following integral equation

$$
\begin{aligned}
\overrightarrow{\boldsymbol{\Phi}}_{\delta}(u)= & \int_{0}^{\infty} \int_{0}^{k} e^{-\delta t} \mathbf{W}^{\prime}(u, t, y) \overrightarrow{\boldsymbol{\Phi}}_{\delta}(0) d y d t \\
& +\int_{0}^{\infty} \int_{k}^{\ell} e^{-\delta t} \mathbf{W}^{\prime}(u, t, y)\left[\int_{0}^{y} e^{-\delta s} f_{L}(s) e^{\mathbf{D}_{0} s} \overrightarrow{\boldsymbol{\Phi}}_{\delta}(s) d s\right. \\
& +\int_{0}^{y} e^{-\delta s} \bar{F}_{L}(s) e^{\mathbf{D}_{0} s} \mathbf{T}_{12} \int_{0}^{\infty} e^{\mathbf{T}_{22} x} \vec{t}_{22} \widetilde{\omega}(s-y, y+x-s) d x d s \\
& \left.+e^{-\delta y} \bar{F}_{L}(y) e^{\mathbf{D}_{0} y} \overrightarrow{\boldsymbol{\Phi}}_{\delta}(0)\right] d y d t \\
& +\int_{0}^{\infty} \int_{0}^{\infty} \int_{\ell}^{\infty} e^{-\delta t} \mathbf{F}^{\prime}(u, t, x, y) \overrightarrow{1}_{m} \widetilde{\omega}(x, y) d y d x d t
\end{aligned}
$$

where $\vec{t}_{22}=-\mathbf{T}_{22} \overrightarrow{1}_{m}$.

Now, let us define the matrices

$$
\mathbf{G}_{\delta}^{\prime}(u, y)=\left(g_{i j}^{\delta}(u, y)\right)_{i, j=1}^{m}=\int_{0}^{\infty} e^{-\delta t} \mathbf{W}^{\prime}(u, t, y) d t
$$

and

$$
\mathbf{G}_{\delta}^{[a, b]}(u)=\left(G_{\delta, i j}^{[a, b]}(u, y)\right)_{i, j=1}^{m}=\int_{a}^{b} \mathbf{G}_{\delta}^{\prime}(u, y) d y,
$$

which denote the joint discounted density function of ruin and the deficit at ruin and the joint discounted probability of ruin with a deficit size in the interval $[a, b]$, respectively. Employing these matrices, the integral (3.1) can be re-written in the form

$$
\overrightarrow{\boldsymbol{\Phi}}_{\delta}(u)=\mathbf{A}_{\delta}(u) \overrightarrow{\boldsymbol{\Phi}}_{\delta}(0)+\vec{b}_{\delta}(u)+\int_{0}^{\ell} \mathbf{G}_{\delta}^{[s \vee k, \ell]}(u) e^{-\delta s} f_{L}(s) e^{\mathbf{D}_{0} s} \overrightarrow{\boldsymbol{\Phi}}_{\delta}(s) d s,
$$

where

$$
\mathbf{A}_{\delta}(u)=\mathbf{G}_{\delta}^{[0, k]}(u)+\int_{k}^{\ell} \mathbf{G}_{\delta}^{\prime}(u, y) e^{-\delta y} \bar{F}_{L}(y) e^{\mathbf{D}_{0} y} d y,
$$

and

$$
\begin{aligned}
\vec{b}_{\delta}(u)= & \int_{k}^{\ell} \mathbf{G}_{\delta}^{\prime}(u, y) \int_{0}^{y} e^{-\delta s} \bar{F}_{L}(s) e^{\mathbf{D}_{0} s} \mathbf{T}_{12} \int_{0}^{\infty} e^{\mathbf{T}_{22} x} \vec{t}_{22} \widetilde{\omega}(s-y, y+x-s) d x d s d y \\
& +\int_{0}^{\infty} \int_{0}^{\infty} \int_{\ell}^{\infty} e^{-\delta t} \mathbf{F}^{\prime}(u, t, x, y) \overrightarrow{1}_{m} \widetilde{\omega}(x, y) d y d x d t
\end{aligned}
$$

where $x \vee y$ denotes the maximum between $x$ and $y$, whilst $\mathbf{F}^{\prime}(u, t, x, y)$ and $\mathbf{G}_{\delta}^{\prime}(u, y)$ are known risk quantities from the MAP/PH risk model in the absence of capital injections.

In order to obtain an explicit solution to the integral (3.4), we first have to determine the constant value $\overrightarrow{\boldsymbol{\Phi}}_{\delta}(0)$, which can be found by setting the initial capital $u=0$ within the integral equation itself and solving the resulting equation with respect to $\overrightarrow{\boldsymbol{\Phi}}_{\delta}(0)$, yielding

$$
\overrightarrow{\boldsymbol{\Phi}}_{\delta}(0)=\left(\mathbf{I}_{m}-\mathbf{A}_{\delta}(0)\right)^{-1}\left[\vec{b}_{\delta}(0)+\int_{0}^{\ell} \mathbf{G}_{\delta}^{[s \vee k, \ell]}(0) e^{-\delta s} f_{L}(s) e^{\mathbf{D}_{0} s} \overrightarrow{\boldsymbol{\Phi}}_{\delta}(s) d s\right],
$$

provided the inverse matrix $\left(\mathbf{I}_{m}-\mathbf{A}_{\delta}(0)\right)^{-1}$ exists, which is proved in the following lemma. 
Lemma 1 Under the net profit condition, the matrix $\left(\mathbf{I}_{m}-\mathbf{A}_{\delta}(0)\right)$ is non-singular and thus invertible.

Proof To show that $\left(\mathbf{I}_{m}-\mathbf{A}_{\delta}(0)\right)$ is a non-singular matrix, by the Lévy-Desplanques Theorem [see Horn and Johnson 2012] it suffices to prove that $\left(\mathbf{I}_{m}-\mathbf{A}_{\delta}(0)\right)$ is a strictly diagonally dominant matrix, i.e.

$$
\begin{aligned}
& \left|1-\left(G_{\delta, i i}^{[0, k]}(0)+\sum_{n=1}^{m} \int_{k}^{\ell} g_{i n}^{\delta}(0, y) e^{-\delta y} \bar{F}_{L}(y)\left(e^{\mathbf{D}_{0} y}\right)_{n i} d y\right)\right| \\
> & \sum_{j \neq i}^{m}\left|G_{\delta, i j}^{[0, k]}(0)+\sum_{n=1}^{m} \int_{k}^{\ell} g_{i n}^{\delta}(0, y) e^{-\delta y} \bar{F}_{L}(y)\left(e^{\mathbf{D}_{0} y}\right)_{n j} d y\right|,
\end{aligned}
$$

for all $i=1, \ldots, m$, or equivalently

$$
1>\sum_{j=1}^{m}\left(G_{\delta, i j}^{[0, k]}(0)+\sum_{n=1}^{m} \int_{k}^{\ell} g_{i n}^{\delta}(0, y) e^{-\delta y} \bar{F}_{L}(y)\left(e^{\mathbf{D}_{0} y}\right)_{n j} d y\right),
$$

since

$$
\begin{aligned}
0 \leqslant & G_{\delta, i i}^{[0, k]}(0)+\sum_{n=1}^{m} \int_{k}^{\ell} g_{i n}^{\delta}(0, y) e^{-\delta y} \bar{F}_{L}(y)\left(e^{\mathbf{D}_{0} y}\right)_{n i} d y \leqslant G_{\delta, i i}^{[0, k]}(0)+\sum_{n=1}^{m} \int_{k}^{\ell} g_{i n}^{\delta}(0, y) d y \\
& \leqslant \sum_{n=1}^{m}\left(G_{\delta, i n}^{[0, k]}(0)+\int_{k}^{\ell} g_{\text {in }}^{\delta}(0, y) d y\right)=\sum_{n=1}^{m} G_{\delta, i n}^{[0, \ell]}(0) \leqslant 1 .
\end{aligned}
$$

Using similar arguments, for the R.H.S. of the inequality in Eq. 3.8, we have

$$
\begin{aligned}
& \sum_{j=1}^{m}\left(G_{\delta, i j}^{[0, k]}(0)+\sum_{n=1}^{m} \int_{k}^{\ell} g_{i n}^{\delta}(0, y) e^{-\delta y} \bar{F}_{L}(y)\left(e^{\mathbf{D}_{0} y}\right)_{n j} d y\right) \\
\leqslant & \left.\sum_{j=1}^{m} G_{\delta, i j}^{[0, k]}(0)+\sum_{j=1}^{m} \sum_{n=1}^{m} \int_{k}^{\ell} g_{i n}^{\delta}(0, y)\left(e^{\mathbf{D}_{0} y}\right)_{n j} d y\right) d y \\
\leqslant & \sum_{j=1}^{m}\left(G_{\delta, i j}^{[0, k]}(0)+\int_{k}^{\ell} g_{i j}^{\delta}(0, y) d y\right)=\sum_{j=1}^{m} G_{\delta, i j}^{[0, \ell]}(0)<1,
\end{aligned}
$$

under the net profit condition, which proves (3.8) and thus completes the proof.

Substituting the explicit form of the boundary condition, $\overrightarrow{\boldsymbol{\Phi}}_{\delta}(0)$, given in Eq. 3.7, back into Eq. 3.4, we get the following proposition.

Proposition 1 For $u \geq 0$, the Gerber-Shiu vector, $\overrightarrow{\boldsymbol{\Phi}}_{\delta}(u)$, satisfies the integral equation

$$
\overrightarrow{\boldsymbol{\Phi}}_{\delta}(u)=\vec{v}_{\delta}(u)+\int_{0}^{\ell} \mathbf{K}_{\delta}(u, s) \overrightarrow{\boldsymbol{\Phi}}_{\delta}(s) d s
$$


which is in the form of an inhomogeneous Fredholm integral equation of the second kind with kernel $\mathbf{K}_{\delta}(u, s)$, given by

$$
\mathbf{K}_{\delta}(u, s)=\mathbf{L}_{\delta}(u, s) f_{L}(s) e^{\left(\mathbf{D}_{0}-\delta \mathbf{I}_{m}\right) s},
$$

where

$$
\vec{v}_{\delta}(u)=\mathbf{A}_{\delta}(u)\left(\mathbf{I}_{m}-\mathbf{A}_{\delta}(0)\right)^{-1} \vec{b}_{\delta}(0)+\vec{b}_{\delta}(u),
$$

and

$$
\mathbf{L}_{\delta}(u, s)=\mathbf{A}_{\delta}(u)\left(\mathbf{I}_{m}-\mathbf{A}_{\delta}(0)\right)^{-1} \mathbf{G}_{\delta}^{[s \vee k, \ell]}(0)+\mathbf{G}_{\delta}^{[s \vee k, \ell]}(u),
$$

with $\mathbf{A}_{\delta}(u)$ and $\vec{b}_{\delta}(u)$, given by Eqs. 3.5 and 3.6, respectively.

\section{An Explicit Solution for the Fredholm Matrix Equation}

In this section, we derive an explicit expression for the Fredholm integral equation system of Proposition 1 and thus, an explicit expression for the Gerber-Shiu function.

Following from the general theory of solving Fredholm integral equations [see Zemyan 2012], the integral (3.9) has an explicit and obtainable solution provided that the kernel, $\mathbf{K}_{\delta}(u, s)$, is separable, by which we mean it can be written in the (general) form

$$
\mathbf{K}_{\delta}(u, s)=\sum_{i=1}^{N} \mathbf{M}_{\delta, i}(u) \mathbf{N}_{\delta, i}(s),
$$

for some matrices $\mathbf{M}_{\delta, i}(u)$ and $\mathbf{N}_{\delta, i}(s)$. It follows from the form of the kernel and the matrix $\mathbf{L}_{\delta}(u, s)$, given in Proposition 1 , that $\mathbf{K}_{\delta}(u, s)$ is separable provided the matrix $\mathbf{G}_{\delta}^{[s, \ell]}(u)$, for $s \leqslant \ell$, is separable.

In order to show the matrix $\mathbf{G}_{\delta}^{[s, \ell]}(u)$ is separable, we recall the analysis of the GerberShiu function for a MAP/PH risk model in the absence of capital injections [see Ahn and Badescu (2007)]. In this paper, the solution of the Gerber-Shiu function avoids the use of roots to the generalised Lundberg's equation and, instead, is represented in terms of the Laplace-Stieltjes transform (LST) of the so-called busy period duration of a fluid flow, which can be represented as

$$
\boldsymbol{\Psi}(\delta)=\int_{0}^{\infty} e^{-\delta x} e^{\Sigma(\delta) x} \mathbf{T}_{12} e^{\mathbf{T}_{22} x} d x
$$

where

$$
\Sigma(\delta)=\left(\mathbf{T}_{11}-\delta \mathbf{I}_{m}\right)+\boldsymbol{\Psi}(\delta) \mathbf{T}_{21},
$$

for which the matrix $\boldsymbol{\Psi}(\delta)$ can be calculated iteratively as a solution to a fixed point problem [see Asmussen (2000), Chapter VIII, Proposition 4.3]. Based on the LST of the busy period in the risk process we obtain the following lemma.

Lemma 2 For $u \geq 0$, the discounted deficit at ruin matrix, $\mathbf{G}_{\delta}^{[s, \ell]}(u)$, is separable and can be written in the form

$$
\mathbf{G}_{\delta}^{[s, \ell]}(u)=\mathbf{M}_{\delta}(u) \mathbf{N}(s)
$$

where

$$
\mathbf{M}_{\delta}(u)=\boldsymbol{\Psi}\left(\frac{\delta}{2}\right) e^{\left[\mathbf{T}_{22}+\mathbf{T}_{21} \boldsymbol{\Psi}\left(\frac{\delta}{2}\right)\right] u} \quad \text { and } \quad \mathbf{N}(s)=\int_{s}^{\ell} e^{\mathbf{T}_{22} y} \mathbf{T}_{21} d y
$$


Proof Setting the deficit dependent penalty function $\omega_{1}(z)=\mathbb{I}_{(z \leq y)}$ in Theorem 4.3 of Ahn and Badescu (2007), replacing the vector $\vec{t}_{22}$ with the matrix $\mathbf{T}_{21}$ to account for the state of the MAP at the time of ruin and differentiating the resulting equation w.r.t. $y$, we obtain

$$
\mathbf{G}_{\delta}^{\prime}(u, y)=\boldsymbol{\Psi}\left(\frac{\delta}{2}\right) e^{\left[\mathbf{T}_{22}+\mathbf{T}_{21} \boldsymbol{\Psi}\left(\frac{\delta}{2}\right)\right] u} e^{\mathbf{T}_{22} y} \mathbf{T}_{21} .
$$

Then, by recalling (3.3), the result follows immediately.

Now, since $\mathbf{G}_{\delta}^{[s, \ell]}(u)$, is separable, it follows that the kernel is itself separable and can be expressed in the form

$$
\mathbf{K}_{\delta}(u, s)=\mathbf{M}_{\delta, 1}(u) \mathbf{N}_{\delta, 1}(s),
$$

where

$$
\mathbf{M}_{\delta, 1}(u)=\mathbf{A}_{\delta}(u)\left(\mathbf{I}_{m}-\mathbf{A}_{\delta}(0)\right)^{-1} \mathbf{M}_{\delta}(0)+\mathbf{M}_{\delta}(u)
$$

and

$$
\mathbf{N}_{\delta, 1}(s)=\mathbf{N}(s \vee k) f_{L}(s) e^{\mathbf{D}_{0} s},
$$

where $\mathbf{N}(s)$ is given by Lemma 2 .

Substituting this form of the kernel back into Eq. 3.9, yields

$$
\overrightarrow{\boldsymbol{\Phi}}_{\delta}(u)=\vec{v}_{\delta}(u)+\mathbf{M}_{\delta, 1}(u) \int_{0}^{\ell} \mathbf{N}_{\delta, 1}(s) \overrightarrow{\boldsymbol{\Phi}}_{\delta}(s) d s,
$$

for which, for smooth choices of $\widetilde{\omega}(\cdot, \cdot)$, the integral $\int_{0}^{\ell} \mathbf{N}_{\delta, 1}(s) \overrightarrow{\boldsymbol{\Phi}}_{\delta}(s) d s$ converges and evaluates to some column vector of constants, say $\vec{\beta}_{\delta}$. Thus, the general solution to the above integral equation is given by

$$
\overrightarrow{\boldsymbol{\Phi}}_{\delta}(u)=\vec{v}_{\delta}(u)+\mathbf{M}_{\delta, 1}(u) \vec{\beta}_{\delta},
$$

and it remains to determine the value of the constant vector $\vec{\beta}_{\delta}$. This can be found following a similar methodology to that of the scalar case in Ramsden and Papaioannou (2019). That is, we multiply (4.3) by $\mathbf{N}_{\delta, 1}(s)$ (on the left) and integrate from 0 to $\ell$, to give

$$
\int_{0}^{\ell} \mathbf{N}_{\delta, 1}(s) \overrightarrow{\boldsymbol{\Phi}}_{\delta}(s) d s=\int_{0}^{\ell} \mathbf{N}_{\delta, 1}(s) \vec{v}_{\delta}(s) d s+\int_{0}^{\ell} \mathbf{N}_{\delta, 1}(s) \mathbf{M}_{\delta, 1}(s) \vec{\beta}_{\delta} d s .
$$

Now, noticing that the integral on the left hand side of the above equation is exactly the vector of constants, $\vec{\beta}_{\delta}$, solving for this vector yields the following theorem.

Theorem 1 For $u \geq 0$, in the MAP/PH risk model with deficit dependent delayed capital injections, the Gerber-Shiu function, $\widetilde{\phi}_{\delta}(u)$, is given by

$$
\widetilde{\phi}_{\delta}(u)=\vec{\alpha}^{\top} \overrightarrow{\boldsymbol{\Phi}}_{\delta}(u),
$$

where $\vec{\alpha}$ is the initial probability vector of the MAP and $\overrightarrow{\boldsymbol{\Phi}}_{\delta}(u)$ is given by

$$
\overrightarrow{\boldsymbol{\Phi}}_{\delta}(u)=\vec{v}_{\delta}(u)+\mathbf{M}_{\delta, 1}(u) \vec{\beta}_{\delta},
$$

with

$$
\vec{\beta}_{\delta}=\left[\mathbf{I}_{m n}-\int_{0}^{\ell} \mathbf{N}_{\delta, 1}(s) \mathbf{M}_{\delta, 1}(s) d s\right]^{-1} \int_{0}^{\ell} \mathbf{N}_{\delta, 1}(s) \vec{v}_{\delta}(s) d s,
$$

and $\vec{v}_{\delta}(u), \mathbf{M}_{\delta, 1}(u)$ and $\mathbf{N}_{\delta, 1}(u)$ are given by Eqs. 3.10, 4.1 and 4.2, respectively. 


\section{The Expected Discounted Accumulated Capital Injections until Ruin}

In this section, we consider a further quantity that will be of interest to an insurance company when it comes to quantitative risk management and mitigation, namely the expected discounted accumulated capital injections up to the time of ultimate ruin, which gives an indication of the (discounted) amount of funds needed to keep the company solvent during its lifetime. This particular quantity could be used to determine the net single premium of a reinsurance contract providing the necessary capital injections, as seen in Pafumi (1998) and Nie et al. (2011). Alternatively, it could be used to determine the present value of dividends to be paid to the companies shareholders, who may contribute to such injections when needed.

Let $\left\{Z_{u}(t)\right\}_{t \geq 0}$ be a pure jump process denoting the accumulated capital injections in a continuous time delayed setting, up to time $t \geqslant 0$, for the risk process $\widetilde{U}_{t}$, with initial capital $u \geqslant 0$. Further, define $\overrightarrow{\mathbf{z}}_{\delta}(u)=\left(\tilde{z}_{\delta, 1}(u), \tilde{z}_{\delta, 2}(u), \ldots, \tilde{z}_{\delta, m}(u)\right)^{\top}$, with

$$
\tilde{z}_{\delta, i}(u)=\mathbb{E}\left[e^{-\delta \widetilde{T}} \widetilde{Z}_{u}(\widetilde{T}) \mid \widetilde{U}_{0}=u, J(0)=i\right], \quad \text { for } \quad i \in E,
$$

where $\delta \geqslant 0$ is the constant discount rate and $\widetilde{T}$ the time of ultimate ruin as defined in Section 2. Then, it follows that the unconditional value for the expected, discounted accumulated capital injections until ruin, denoted $\tilde{z}_{\delta}(u)$, is determined by

$$
\tilde{z}_{\delta}(u)=\mathbb{E}\left[e^{-\delta \widetilde{T}} \widetilde{Z}_{u}(\widetilde{T})\right]=\sum_{i=1}^{m} \alpha_{i} \tilde{z}_{\delta, i}(u) .
$$

Given that the MAP process has initial state $i \in E$ and is in state $j \in E$ at the time of deficit, by conditioning on the time and amount of the first fall into deficit, the subsequent delay time, claim inter-arrival time and using the joint density matrix of the time to and size of the deficit, i.e. $\mathbf{W}^{\prime}(u, t, y)$ (defined in Section 2), along with matrices $\mathbf{G}_{\delta}^{\prime}(u, y)=$ $\int_{0}^{\infty} e^{-\delta t} \mathbf{W}^{\prime}(u, t, y) d t$ and $\mathbf{G}_{\delta}^{[a, b]}(u)=\int_{a}^{b} \mathbf{G}_{\delta}^{\prime}(u, y) d y$, we obtain that

$$
\begin{aligned}
\overrightarrow{\mathbf{z}}_{\delta}(u)= & \int_{0}^{\infty} \int_{0}^{k} e^{-\delta t} \mathbf{W}^{\prime}(u, t, y)\left[y \overrightarrow{1}_{m}+\overrightarrow{\mathbf{z}}_{\delta}(0)\right] d y d t+\int_{0}^{\infty} \int_{k}^{\ell} e^{-\delta t} \mathbf{W}^{\prime}(u, t, y) \\
& \times\left[\int_{0}^{y} e^{-\delta s} f_{L}(s) e^{\mathbf{D}_{0} s}\left[y \overrightarrow{1}_{m}+\overrightarrow{\mathbf{z}}_{\delta}(s)\right] d s+e^{-\delta y} \bar{F}_{L}(y) e^{\mathbf{D}_{0} y} \overrightarrow{\mathbf{z}}_{\delta}(0)\right] d y d t \\
= & \int_{0}^{k} y \mathbf{G}_{\delta}^{\prime}(u, y) \overrightarrow{1}_{m} d y+\mathbf{G}_{\delta}^{[0, k]}(u) \overrightarrow{\mathbf{z}}_{\delta}(0)+\int_{k}^{\ell} \bar{F}_{L}(y) \mathbf{G}_{\delta}^{\prime}(u, y) e^{-\left[\delta \mathbf{I}_{m}-\mathbf{D}_{0}\right] y} \overrightarrow{\mathbf{z}}_{\delta}(0) d y \\
& +\int_{k}^{\ell} y \mathbf{G}_{\delta}^{\prime}(u, y) \int_{0}^{y} f_{L}(s) e^{-\left[\delta \mathbf{I}_{m}-\mathbf{D}_{0}\right] s} \overrightarrow{1}_{m} d s d y \\
& +\int_{0}^{\ell} f_{L}(s) \mathbf{G}_{\delta}^{[s \vee k, \ell]}(u) e^{-\left[\delta \boldsymbol{I}_{m}-\mathbf{D}_{0}\right] s} \overrightarrow{\mathbf{z}}_{\delta}(s) d s \\
= & \vec{\chi}_{\delta}(u)+\mathbf{A}_{\delta}(u) \overrightarrow{\mathbf{z}}_{\delta}(0)+\int_{0}^{\ell} f_{L}(s) \mathbf{G}_{\delta}^{[s \vee k, \ell]}(u) e^{-\left[\delta \boldsymbol{I}_{m}-\mathbf{D}_{0}\right] s} \overrightarrow{\mathbf{z}}_{\delta}(s) d s,
\end{aligned}
$$

where, $\mathbf{A}_{\delta}(u)$ is given by Eq. 3.5 and

$$
\vec{\chi}_{\delta}(u)=\int_{0}^{k} y \mathbf{G}_{\delta}^{\prime}(u, y) \overrightarrow{1}_{m} d y+\int_{k}^{\ell} y \mathbf{G}_{\delta}^{\prime}(u, y) \int_{0}^{y} f_{L}(s) e^{-\left[\delta \boldsymbol{I}_{m}-\mathbf{D}_{0}\right] s} \overrightarrow{1}_{m} d s d y .
$$


In a similar way to the previous sections, in order to find an explicit solution to the integral (5.1), we first have to determine $\overrightarrow{\mathbf{z}}_{\delta}(0)$, which can be found by setting $u=0$ in Eq. 5.1 and then solving with respect to $\overrightarrow{\mathbf{z}}_{\delta}(0)$, which gives

$$
\overrightarrow{\mathbf{z}}_{\delta}(0)=\left(\boldsymbol{I}_{m}-\mathbf{A}_{\delta}(0)\right)^{-1}\left[\vec{\chi}_{\delta}(0)+\int_{0}^{\ell} f_{L}(s) \mathbf{G}_{\delta}^{[s \vee k, \ell]}(0) e^{-\left[\delta \boldsymbol{I}_{m}-\mathbf{D}_{0}\right] s} \overrightarrow{\mathbf{z}}_{\delta}(s) d s\right],
$$

where $\left(\boldsymbol{I}_{m}-\mathbf{A}_{\delta}(0)\right)^{-1}$ exists by Lemma 1 . Then, by substituting the expression for $\overrightarrow{\mathbf{z}}_{\delta}(0)$ in Eq. 5.1, we have the following proposition.

Proposition 2 For $u \geq 0$, the expected discounted accumulated capital injections up to the time of ultimate ruin, $\overrightarrow{\mathbf{z}}_{\delta}(u)$, satisfies the integral equation

$$
\overrightarrow{\mathbf{z}}_{\delta}(u)=\vec{\zeta}_{\delta}(u)+\int_{0}^{\ell} \mathbf{K}_{\delta}(u, s) \overrightarrow{\mathbf{z}}_{\delta}(s) d s,
$$

which is in the form of an inhomogeneous Fredholm integral equation of the second kind with kernel $\mathbf{K}_{\delta}(u, s)$ given by Proposition 1 and

$$
\vec{\zeta}_{\delta}(u)=\boldsymbol{A}_{\delta}(u)\left(\boldsymbol{I}_{m}-\boldsymbol{A}_{\delta}(0)\right)^{-1} \vec{\chi}_{\delta}(0)+\vec{\chi}_{\delta}(u),
$$

with $\vec{\chi}_{\delta}(u)$ given by Eq. 5.2.

Now recall from Section 4 that $\mathbf{K}_{\delta}(u, s)$ is separable, i.e. $\mathbf{K}_{\delta}(u, s)=\mathbf{M}_{\delta, 1}(u) \mathbf{N}_{\delta, 1}(s)$, where $\mathbf{M}_{\delta, 1}(u)$ and $\mathbf{N}_{\delta, 1}(s)$ are given by Eqs. 4.1 and 4.2, respectively and thus adopting the same methodology as in Section 4, the explicit solution to the inhomogeneous Fredholm integral equation of the second kind in Eq. 5.3 is given by the following theorem.

Theorem 2 For $u \geq 0$, in the MAP/PH risk model with deficit dependent delayed capital injections, the expected discounted capital injections until ruin, is given by

$$
\tilde{z}_{\delta}(u)=\vec{\alpha}^{\top} \overrightarrow{\mathbf{z}}_{\delta}(u),
$$

where $\vec{\alpha}$ is the initial probability vector of the MAP and $\overrightarrow{\mathbf{z}}_{\delta}(u)$ is given by

$$
\overrightarrow{\mathbf{z}}_{\delta}(u)=\vec{\zeta}_{\delta}(u)+\mathbf{M}_{\delta, 1}(u) \vec{\gamma}_{\delta}
$$

with

$$
\vec{\gamma}_{\delta}=\left[\mathbf{I}_{m n}-\int_{0}^{\ell} \mathbf{N}_{\delta, 1}(s) \mathbf{M}_{\delta, 1}(s) d s\right]^{-1} \int_{0}^{\ell} \mathbf{N}_{\delta, 1}(s) \vec{\zeta}_{\delta}(s) d s
$$

and $\vec{\zeta}_{\delta}(u), \mathbf{M}_{\delta, 1}(u)$ and $\mathbf{N}_{\delta, 1}(u)$ given by Eqs. 5.4, 4.1 and 4.2, respectively.

\section{Numerical Illustration}

In the previous sections, we have shown that a number of risk quantities can be expressed explicitly for the MAP/PH delayed capital injection risk model. Although these expressions are indeed explicit, they are quite complex and require a number of substitutions and matrix manipulations. Therefore, in this section, we illustrate the applicability and emphasise the accessibility of these results numerically by providing examples for a particular case of the Gerber-Shiu function, namely the ruin probability, as well as, examples for the expected accumulated capital injections until ruin.

For comparison purposes, we choose the same parameters as in Ahn and Badescu (2007). That is, the inter-arrival times governed by the MAP, follow a generalised Erlang distribution 
having three phases with rates $\lambda_{1}=\lambda_{2}=0.5$ and $\lambda_{3}=0.3$, respectively. Moreover, the initial probability vector is given by $\alpha=(1,0,0)$, so that the mean claim arrival time is 7.33. Moreover, the non-dependent claims (independent of claim arrival transition states see Remark 1) follow the Phase-type distribution with representation

$$
\vec{\gamma}^{\top}=(0.1,0.1,0.3,0.5)
$$

and

$$
\mathbf{H}=\left(\begin{array}{cccc}
-1 / 10 & 1 / 10 & 0 & 0 \\
0 & -1 / 6 & 1 / 6 & 0 \\
0 & 0 & -1 / 3 & 1 / 3 \\
0 & 0 & 0 & -1 / 2
\end{array}\right)
$$

For the delay clock random variable $L$, we consider three different distributions for illustration and comparative purposes:

(i) Exponential Delay (ExD) clock with mean 3.32,

(ii) An Erlang Delay (ErD) clock with mean 7.33, initial probability vector $(1,0,0)$ and generator matrix

$$
\left(\begin{array}{ccc}
-0.4928 & 0.4928 & 0 \\
0 & -0.4928 & 0.4928 \\
0 & 0 & -0.4928
\end{array}\right),
$$

(iii) A Generalised Erlang Delay (GErD) clock with mean 16.89, initial probability vector $(1,0,0)$ and the generator matrix

$$
\left(\begin{array}{ccc}
-1.5 & 1.5 & 0 \\
0 & -0.17763 & 0.17763 \\
0 & 0 & -0.09440
\end{array}\right) .
$$

Finally, the first and the second critical values of the risk process $\widetilde{U}_{t}$ are chosen to be $k=0.5$ and $\ell=10$, respectively.

\subsection{Ruin Probabilities}

In this subsection, we provide numerical results for the ruin probabilities. That is, we set $\delta=0$ and $\widetilde{\omega}(\cdot, \cdot)=1$, which reduces the Gerber-Shiu function in Eq. 2.3 to the well known ruin probability.

Based on the above set of parameters the numerics for ruin probabilities, given the initial starting phase of the inter-arrival time distribution, for the ExD, ErD and GErD clocks are given by the table below.

Remark 3 We observe from the table above that the ruin probabilities increase as the expected delay time increases. This result is expected due to the definition of ruin in the above model as the longer the insurance firm has to wait for the required capital injection, whilst in red, the more probable is its for the insurance firm to inccur a subsequent claim and cause ultimate ruin, see also Figs. 2, 3, 4 and Table 1.

For a graphical representation of these results, the probability of ruin for the aforementioned three delay clocks (as a function of the initial surplus) have also been plotted in the figures below. 


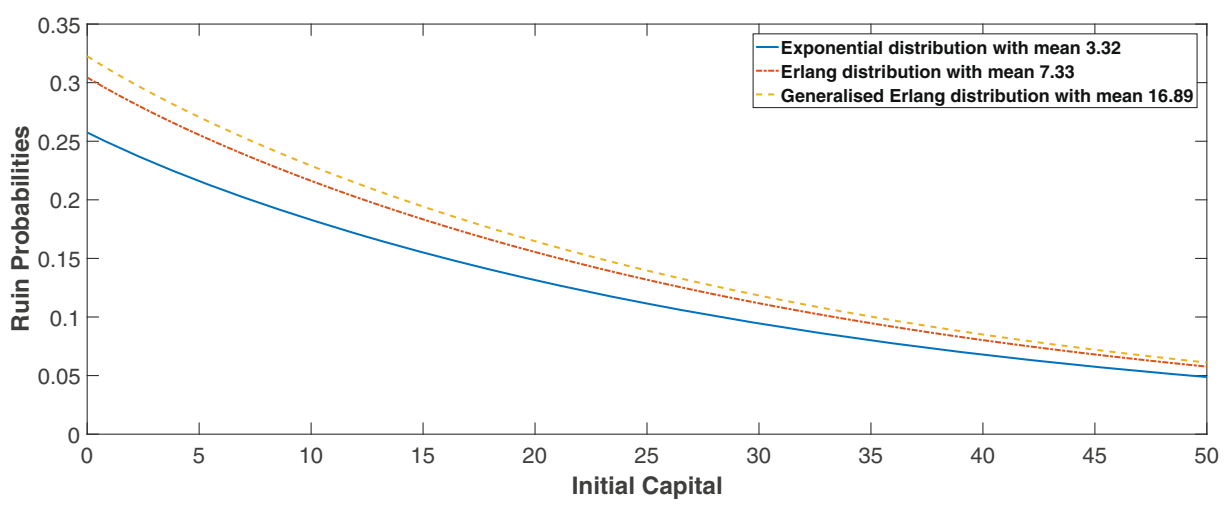

Fig. 2 Initial Phase I: Ruin Probabilities vs Initial Capital

\subsection{The Expected Discounted Accumulated Capital Injections}

In this subsection, we also provide some numerical results for the expected accumulated capital injections until ruin.

Based on the above set of parameters and the initial starting phase of the inter-arrival time distribution, we present below the numerical results for the expected accumulated capital injections until ruin ExD, ErD and GErD clocks. Note that these results correspond to the un-discounted injections as we have set the parameter $\delta=0$.

Graphically the above results (as a function of the initial surplus) are given by the figures below.

Remark 4 We observe from these results that the expected accumulated capital injections decrease as the expected delay time increases. This should be expected due to the fact that a longer delay time before receipt of the required capital injection exposes the firm to a higher chance of ruin due to a subsequent claim arrival when in deficit (See Figs. 5, 6 and

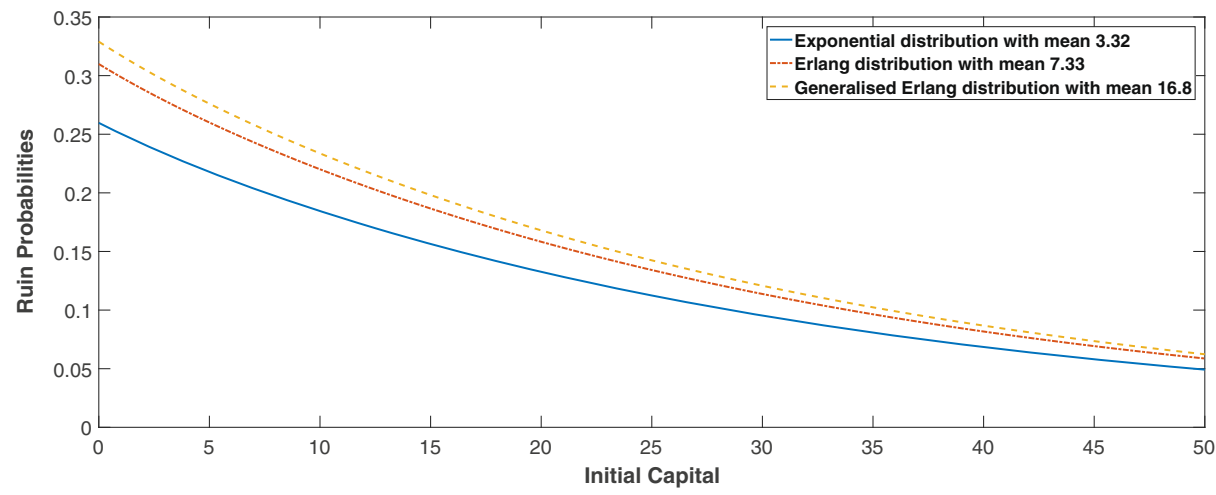

Fig. 3 Initial Phase II: Ruin Probabilities vs Initial Capital 


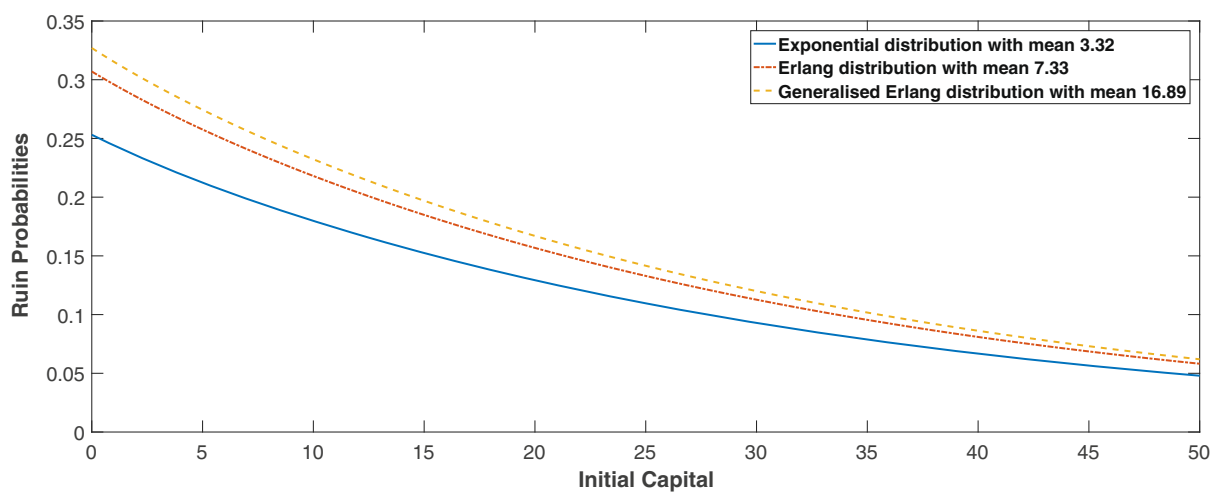

Fig. 4 Initial Phase III: Ruin Probabilities vs Initial Capital

Table 1 Ruin Probabilities for Exponential Delay (ExD), Erlang Delay (ErD) and Generalised Erlang Delay (GErD) clocks

\begin{tabular}{|c|c|c|c|c|c|c|c|c|c|}
\hline \multirow[t]{2}{*}{$u$} & \multicolumn{3}{|c|}{ Initial Phase I } & \multicolumn{3}{|c|}{ Initial Phase II } & \multicolumn{3}{|c|}{ Initial Phase III } \\
\hline & ExD & ErD & GErD & ExD & ErD & GErD & ExD & ErD & GErD \\
\hline 0 & 0.2575 & 0.3044 & 0.3225 & 0.2597 & 0.3099 & 0.3290 & 0.2531 & 0.3070 & 0.3270 \\
\hline 10 & 0.1829 & 0.2162 & 0.2291 & 0.1845 & 0.2201 & 0.2336 & 0.1798 & 0.2180 & 0.2322 \\
\hline 30 & 0.0945 & 0.1117 & 0.1183 & 0.0953 & 0.1137 & 0.1207 & 0.0929 & 0.1127 & 0.1200 \\
\hline 50 & 0.0488 & 0.0577 & 0.0611 & 0.0492 & 0.0587 & 0.0623 & 0.0479 & 0.0582 & 0.0619 \\
\hline 100 & 0.0093 & 0.0110 & 0.0117 & 0.0094 & 0.0112 & 0.0119 & 0.0092 & 0.0111 & 0.0118 \\
\hline
\end{tabular}

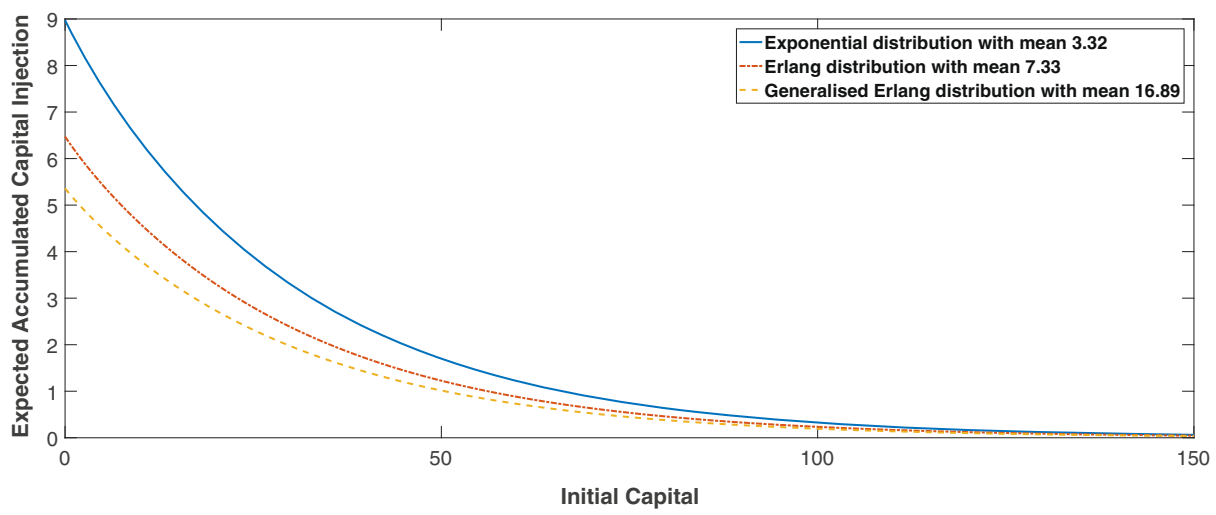

Fig. 5 Initial Phase I: Expected Accumulated Capital Injection vs Initial Capital 


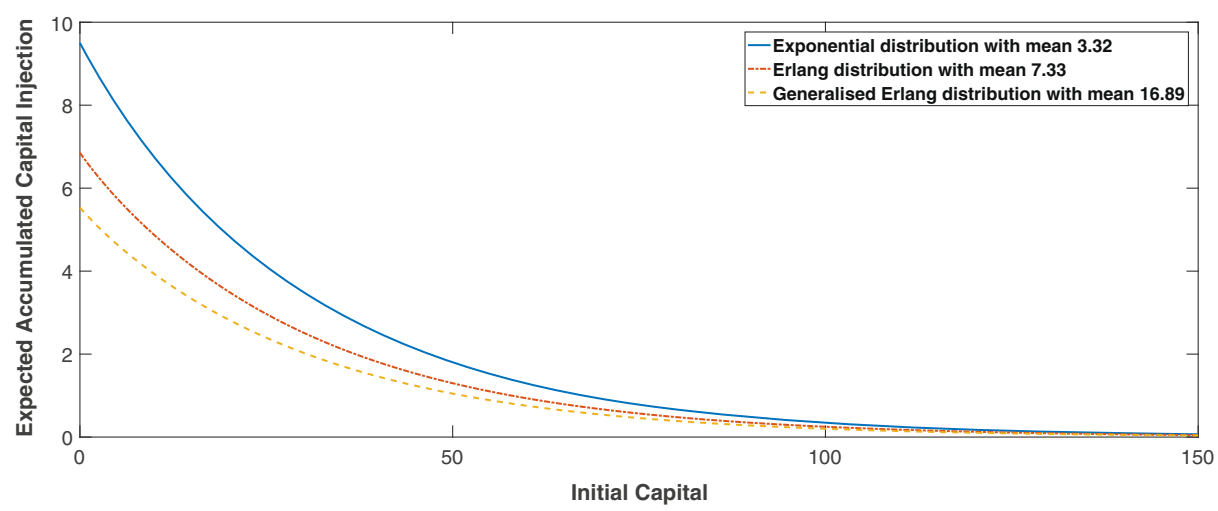

Fig. 6 Initial Phase II: Expected Accumulated Capital Injection vs Initial Capital

7 and Table 2) and consequently, the capital injection is never received. On the other hand, shorter delay times result in a higher probability of the capital injection being received before ultimate ruin and thus allowing the company to continue with the possibility of further injections required for future falls into deficit.

Remark 5 Comparing the numerical results of both of these quantities (ruin probability and expected accumulated capital injections) produces an interesting question of risk 'trade off' for the company. That is, although shorter delay times for the capital injections reduces the probability of ruin, at the same time this causes an increase in the expected accumulated capital injections which have to be considered as a cost to the company, i.e.

Smaller the delay in capital injections $\Rightarrow$ Smaller the ruin probabilities $\Rightarrow$ Bigger the cost of capital (expected accumulated capital injections).

This cost is most likely to come in the form of dividend payments if the injections are funded by the shareholders, returns on equity investment from a government injection or simply a repayment of the injection if received as a loan. In any case, these extra 'costs'

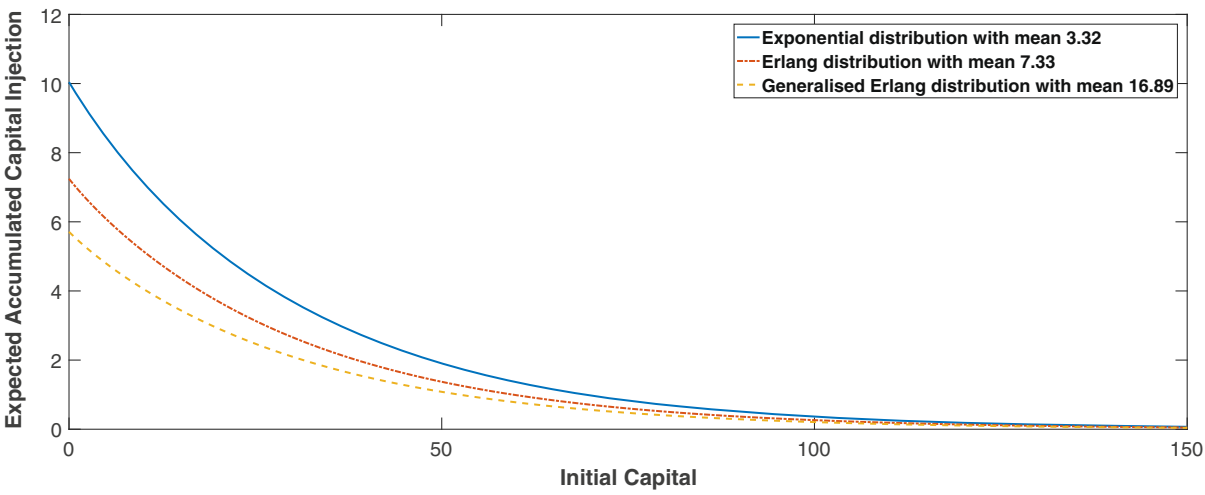

Fig. 7 Initial Phase III: Expected Accumulated Capital Injection vs Initial Capital 
Table 2 Expected accumulated capital injection for Exponential Delay (ExD), Erlang Delay (ErD) and Generalised Erlang Delay (GErD) clock

\begin{tabular}{|c|c|c|c|c|c|c|c|c|c|}
\hline \multirow[t]{2}{*}{$u$} & \multicolumn{3}{|c|}{ Initial Phase I } & \multicolumn{3}{|c|}{ Initial Phase II } & \multicolumn{3}{|c|}{ Initial Phase III } \\
\hline & ExD & ErD & GErD & ExD & ErD & GErD & ExD & ErD & GErD \\
\hline 0 & 8.9729 & 6.4727 & 5.3588 & 9.5003 & 6.8531 & 5.5324 & 10.0423 & 7.2441 & 5.7108 \\
\hline 10 & 6.3729 & 4.5971 & 3.8060 & 6.7475 & 4.8673 & 3.9293 & 7.1324 & 5.1450 & 4.0560 \\
\hline 30 & 3.2933 & 2.3756 & 1.9669 & 3.4869 & 2.5153 & 2.0306 & 3.6858 & 2.6588 & 2.0960 \\
\hline 50 & 1.6997 & 1.2261 & 1.0151 & 1.7996 & 1.2982 & 1.0480 & 1.9023 & 1.3722 & 1.0818 \\
\hline 100 & 0.3251 & 0.2345 & 0.1942 & 0.3442 & 0.2483 & 0.2004 & 0.3638 & 0.2625 & 0.2070 \\
\hline
\end{tabular}

need to be accounted for and will result in a higher probability of ruin to those stated above. Determining the most efficient trade-off is a matter of optimisation and was considered in the non-delayed setting by Nie et al. (2011).

Acknowledgments The authors are grateful to the anonymous referees for their constructive comments and suggestions that have improved the content and presentation of this paper.

Open Access This article is licensed under a Creative Commons Attribution 4.0 International License, which permits use, sharing, adaptation, distribution and reproduction in any medium or format, as long as you give appropriate credit to the original author(s) and the source, provide a link to the Creative Commons licence, and indicate if changes were made. The images or other third party material in this article are included in the article's Creative Commons licence, unless indicated otherwise in a credit line to the material. If material is not included in the article's Creative Commons licence and your intended use is not permitted by statutory regulation or exceeds the permitted use, you will need to obtain permission directly from the copyright holder. To view a copy of this licence, visit http://creativecommonshorg/licenses/by/4.0/.

\section{References}

Asmussen S (2000) Ruin probabilities. World Scientific Second Edition, Singapore

Ahn S, Badescu A (2007) On the analysis of the Gerber-Shiu discounted penalty function for risk processes with Markovian arrivals. Insur Math Econ 41(2):234-239

Badescu A, Breuer L, da Silva Soares A, Latouche G, Remiche M, Stanford D (2005a) Risk processes analyzed as fluid queues. Scand Actuar J 2:127-141

Badescu A, Breuer L, Drekic S, Latouche G, Stanford D (2005b) The surplus prior to ruin and the deficit at ruin for a correlated risk process. Scand Actuar J 6:127-141

Badescu A, Drekic S, Landriault D (2007b) On the Analysis of a Multi-Threshold Markovian Risk Model. Scand Actuarial J 4:248-260

Cheung E, Landriault D (2009) Perturbed MAP risk models with dividend barrier strategies. J Appl Probab 46:521-541

Dibu AS, Jacob MJ (2019) On the Gerber-Shiu function of a MAP risk model with possible delayed phasetype by-claims. preprint

Gerber H, Shiu E (1998) On the time value of ruin. North Amer Actuar J 2:48-78

Horn RA, Johnson CR (2012) Matrix analysis. Cambridge University Press

Kulenko N, Schmidli H (2008) Optimal dividend strategies in a Cramé,r-Lundberg model with capital injections. Insur Math Econ 43(2):270-278

Latouche G, Ramaswami V (1999) Introduction to matrix analytic methods in stochastic modelling. ASASIAM Series on Statistics and Applied Probability , Philadelphia

Neuts MF (1979) A versatile Markovian point process. J Appl Probab 16(4):764-779

Nie C, Dickson DC, Li S (2011) Minimizing the ruin probability through capital injections. Ann Actuar Sci 5(02)):195-209 
Pafumi G (1998) On the time value to ruin. Discuss North Amer Actuar J 2(1):75-76

Ramaswami V (2006) Passage times in fluid models with application to risk processes. Methodol Comput Appl Probab 8(4):497-515

Ramsden L, Papaioannou A (2019) On the time to ruin for a dependent delayed capital injection risk model. Appl Math Comput 352:119-135

Zemyan SM (2012) The classical theory of integral equations: a concise treatment. Springer Science \& Business Media

Zhang Z, Cheung ECK, Yang H (2018) On the compound Poisson risk model with periodic capital injections. ASTIN Bullet 48(1):435-477

Publisher's Note Springer Nature remains neutral with regard to jurisdictional claims in published maps and institutional affiliations. 